\title{
Optimal Control for Smokescreen Release Based on Numerical Simulation: Challenges and Theoretical Framework
}

\author{
Cheng Cui, Sheng Xu, Zhihua Zhu, Feng Liu, Xuezheng Zhu, Shunxiang Huang* \\ Institute of Chemical Defense, Beijing, China \\ Email: shunxianghuang@163.com
}

Received 7 February 2015; accepted 25 March 2015; published 31 March 2015

Copyright (C) 2015 by authors and Scientific Research Publishing Inc.

This work is licensed under the Creative Commons Attribution International License (CC BY).

http://creativecommons.org/licenses/by/4.0/

(c) (i) Open Access

\begin{abstract}
This paper analyses the key issues and challenges of the smokescreen release optimal control under complex terrains and meteorological conditions, and proposes a dynamic model based theoretical framework that integrates the smoke release equipments, the release time, the release positions, different ways to release smoke and launch attacks, various terrains and meteorological conditions and other factors so as to determine the appropriate smokescreen release plans through the rapid inverse for various threats and battlefield environments and to conduct realtime simulation and evaluation. This paper provides a theoretical basis for the smokescreen release optimal control.
\end{abstract}

\section{Keywords}

Numerical Simulation, Atmospheric Dispersion, Smokescreen, Optimal Control

\section{Introduction}

Smokescreen plays an important role on the battlefield with two fundamental functions: disturbance and disguise. Since all the terminal guidance systems rely on electromagnetic waves to realize precision strikes and smokescreen can selectively disturb the electromagnetic waves, it is possible to prevent precision strikes by smokescreen. Smokescreen can disguise visible light, infrared ray, millimeter waves and other wavebands through the shielding effect and thus defend against multiple space-based and ground-based means of reconnaissance [1]. The core issues for using smokescreen are the time, positions and ways of smoke release.

Currently, although some smokescreen estimation models have been developed [1], they are far from meeting the requirements of precision control in the information age and mostly it is estimated by the classical Gauss

\footnotetext{
${ }^{*}$ Corresponding author.
}

How to cite this paper: Cui, C., Xu, S., Zhu, Z.H., Liu, F., Zhu, X.Z. and Huang, S.X. (2015) Optimal Control for Smokescreen Release Based on Numerical Simulation: Challenges and Theoretical Framework. Advances in Chemical Engineering and Science, 5, 158-163. http://dx.doi.org/10.4236/aces.2015.52017 
model. In fact, using smokescreen is essentially an inverse problem of atmospheric diffusion. Usually, we estimate the smoke release effect by simulating the diffusion process of the smoke screens of specified source intensity, positions and time in the atmosphere in order to determine the intensity, amount and shielding effect distribution. However, in the actual situation, our goals are often conduct disturbance or disguise for specified objects at specified time, and the question is how to determine the ways, time and positions of smoke release so as to achieve the above goals. Obviously, this is an inverse question; that is to say, start from the objectives and backward simulate the diffusion process to determine the smoke release plan. On one hand, the Gauss model only applies commonly to the simulation of diffusion on the smooth ground and even underlying surface, while the actual battlefields often have complex terrains and uneven underlying surface. On the other hand, smoke release requires backward simulation starting from the objectives. Apparently, the existing smoke release theories and methods can hardly meet the demands of actual combats. Therefore, it is urgent to take various kinds of threats into account and develop the dynamic models based on the types of the smoke generating equipment, time, positions and ways of smoke release as well as different ways of attack and different terrains and meteorological conditions to inverse the smoke release plans, and thus further break through the bottleneck in smoke protection precision control technologies by providing key support to the rapid calculation of smokescreen protection plans as to different threats. And we should realize the smoke release precision control based on threats and effect estimation, provide methods for the performance evaluation and simulation of smoke combat, avoid aimless smoke release and promote the precision-oriented development of smokescreen calculation and performance evaluation so as to improve the smoke combat ability.

Another critical factor affecting the operational effectiveness of smoke release is to develop and solve rapidly the optimal control model for smoke release. As the battlefield is volatile, once the object is located, smoke release must be rapidly implemented so as to realize the purposes of operation. And in order to determine the release plan and performance rapidly, the efficiency of the diffusion simulation is crucial. Therefore, the priorities are the models with high calculation efficiency including the Lagrangian trajectory puff model [2], the stage smoke plume model or the random walk particle model [3]. Many research achievements have been applied for nuclear emergency, such as the Real-time On-line Decision Support system for nuclear emergency in European (RODOS) [4], and the Lagranian Puff Model adopted by the Chinese Tian Wan Nuclear Power Emergency System [5]. And in order to cope with the chemical emergencies in urban areas, the U.S. launched a large-scale research project under the title of CBNP at the end of last century to study from the perspective of scientific research the surface layer wind field and diffusion dispersion characteristics on the complex urban underlying surfaces through observation experiments and the numerical simulation methods [6]. As a main research method of CBNP, numerical simulation plays an important role in describing the wind field changes of various scales diffusion in urban areas. Regarding the operational system development, there are some successful cases in other countries: the United States developed the Atmospheric Release Advisory Capability (ARAC) [7], and Japan developed the System for Prediction of Environment Emergency Dose Information (SPEEDI) [8]. And we proposed to use the meteorological principles to establish hazard assessment models in cases of chemical terrorist attacks, pointed out the pivotal role that the numerical simulation method should play in responding to chemical emergencies, and explored the theoretical framework and experimental results of the Concentration and Dose Model or the toxic Clouds Diffusion Model over complex terrains (CDM) [3], which could calculate the hazard scope, grade, area, depth and other crucial technical statistics of atmospheric contamination chemical accidents within several minutes so as to provide instant, accurate, dynamic and smart support to the decision-making process of responding to chemical emergencies and had been applied in the Beijing Olympic Games [9], the combat with NBC terrorism [10], the destruction of Japanese Abandoned Chemical Weapons (JACWs) [11], and so on. In terms of the source item inversion method, a lot of research has been done on nuclear chemical accidents [12] [13]. By applying the adjoint principle, we successively developed the optimal control of nuclear-biological-chemical threats, the optimized layout method of industrial pollution sources, the optimal control of air pollution, the selection of plants to destroy the JACWs, the hazard assessment of the chemical agents leakage, and so on [14]-[17]. However, no public report has been made on the smokescreen release precision control. Under such background, this paper proposes a theoretical framework for the smokescreen release precision control.

\section{The Theoretical Framework for the Smokescreen Release Precision Control}

The theoretical framework for the smokescreen release precision control is shown is Figure 1. The results of the 


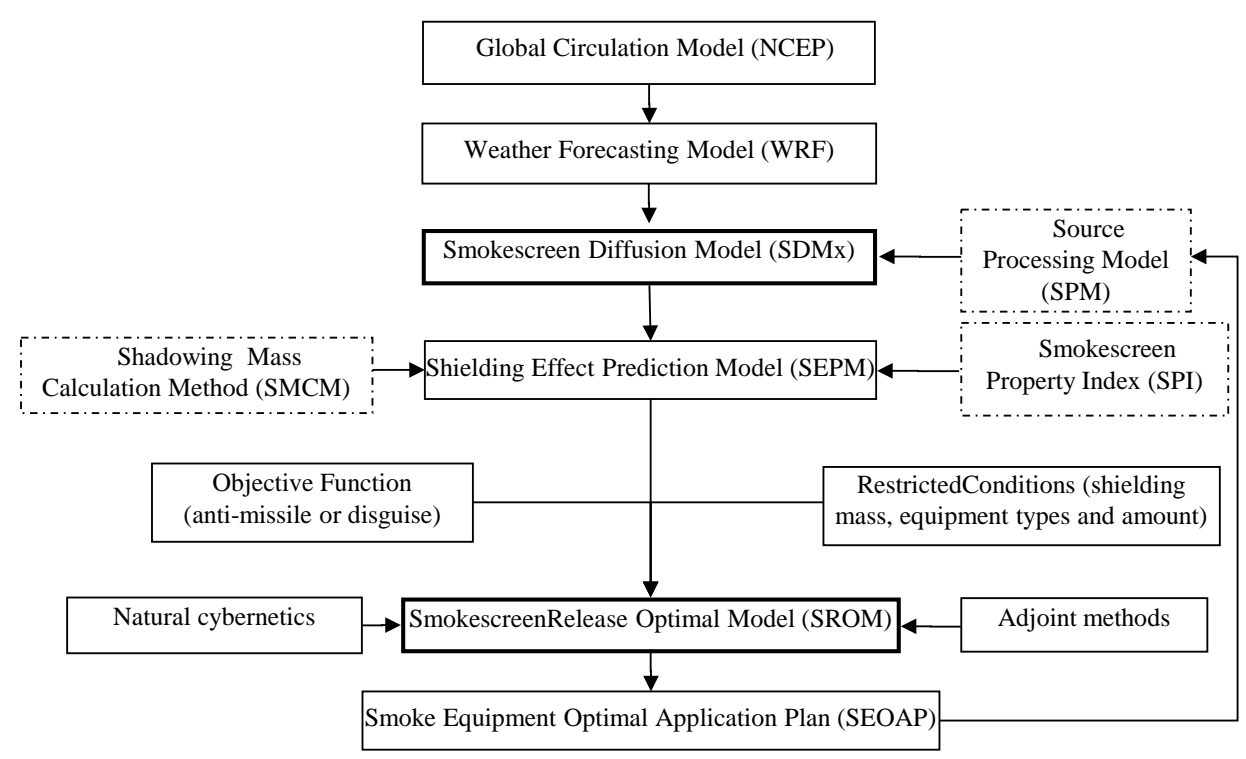

Figure 1. The overall technical route of the smokescreen release precision control.

global circulation model (NCEP) are used as the background fields to run weather forecasting model (WRF) to determine the accurate and elaborate meteorological fields for the target area. The meteorological field from WRF runs the Smokescreen Diffusion Model over complex terrains (SDMx) and at the same time inputs the boundary conditions and source based on the Source Processing Model (SPM) to simulate the smoke diffusion process. And by applying the Smokescreen Property Index (SPI) and the Shadowing Mass Calculation Method (SMCM), the smoke shielding effect is predicted by using the Shielding Effect Prediction Model (SEPM). And based on different operational missions and conditions of the battlefield, determine the objective function (the shielding scope, period, place, and so on) to be protected (disguised or from missiles). And depending on the types, amount and smoke shielding massy of smokescreen release equipments, fix the restricted conditions to conduct the smokescreen protection.

While the results of the SDMx run the Smokescreen Release Optimal Model (SROM), the SROM adopts the natural cybernetics and the adjoint method to optimize the equipment placement and the smoke release and further determine the optimal equipment application plan [18]. During the optimization, the equipment application plan and the shielding effect feedback to each other, and through distributed computation, the sources determined by the equipment application plan is integrated to the diffusion model and then the renewed smoke release plan's shielding mass is worked out to obtain the Smoke Equipment Optimal Application Plan (SEOAP).

The entire optimal control process is a complex system that reflects the actual situation of using smokescreen in the battlefield. Based on principles of dynamics and economics, it takes into account not only the constraints including the operation troops, equipments and technologies but also the shielding requirements of the objects to be protected. And it adopts the objective function with the lowest overall costs for the equipment application to work out the optimal real-time smoke protection plan.

Meteorological fields forecasting. The operational environment is very complex, affected by both the systematic winds formed as a result of the global circulation and the local circulation. And the dynamic and thermodynamic activities of the terrains and the uneven underlying surfaces determine the formation, development and dissipation of the local circulations. Therefore, it's necessary to study the precision forecast of the typical meteorological fields that may occur on the battlefield, select the proper meteorological forecasting models and parameterization schemes, and conduct research on the sea-and-land breezes, the up valley winds, the lake-andland breezes and the urban heat island effects based on our operational mission. And apply the scientific research data to developing technology so as to improve the accuracy of meteorological forecasting. Use the background fields from the global circulation model in the regional meteorological forecasting model and use the multi-layer nesting technology to conduct accurate forecast of the meteorological fields $M f\left(u, v, w, t, e, K_{h}, K_{v}\right)$ of the target area. Among them, $u, v, w$ are the three dimensional wind speeds; $t$ stands for temperature and e stands for turbulent kinetic energy; and represent horizontal and vertical diffusion coefficients respectively. 
Diffusion simulation. Adopt the Euler theory or the Langrage theory to establish the Smokescreen Diffusion Model over complex terrains (SDMx). Conduct comparative studies of field diffusion experiments, physical modeling experiments (wind tunnel experiments and flume experiments) and numerical models to verify the applicability and accuracy of the smokescreen diffusion model. Study the coupling technology of the smokescreen diffusion model and the regional meteorological field forecasting model, use the latter to work out the delicate meteorological field to further run the SDMx which at the same time inputs the boundary conditions and source item to simulate the smokescreen (aerosol) diffusion process and the spatial and temporal distribution of the shielding mass $[M b(x, y, z ; t)]$ in the target area. And $M b$ refers to

$$
M b(x, y, z ; t)=\int_{l_{1}\left(x_{1}, y_{1}, z_{1}\right)}^{l_{2}\left(x_{2}, y_{2}, z_{2}\right)} c(x, y, z ; t) \mathrm{d} l
$$

In Equation (1), $l_{1}\left(x_{1}, y_{1}, z\right)_{1}$ and $l_{2}\left(x_{2}, y_{2}, z_{2}\right)$ are the places of the protected and shielded objects respectively.

Smokescreen release optimal model. Smokescreen diffusion is a question of natural science while smoke release is controlled by human activities. And the two are well connected by the natural cybernetics. Therefore, the natural cybernetics should be used to establish the Smokescreen Release Optimal Model (SROM).

Objective functions. By collecting information on the tactical and technical performances of various air raid weapons in the hands of the potential opponents, familiarize ourselves with the common air raid methods and the guidance models of the air raid weapons; by summarizing the tactical and technical performance parameters of our smokescreen release equipments and different smokescreen protection methods, clarify the smokescreen protection procedures and requirements; and by collecting materials on air situation information sharing, interactive mechanisms and methods and technology of our air defense system, determine the shielding mass and the shielding areas and promote the organic integration of the air situation information and the smokescreen release. The types and amount of smoke release equipments in military service can be determined only on the basis of studying the relationship between the smokescreen protection, threats and objects to be protected, and thus provides essential data for the determination of the objective function. Suppose $E k$ stands for the type of the smoke release equipments, $E q$ for the amount of the smoke release equipments, and then the objective function of the smoke protection plan

$$
J=G(E k, E q)
$$

When the shielding mass in the protected area just exceeds some certain threshold value $M b_{0}$, the objective function $J$ in connection with the types and amount of the smokescreen release equipments will be the lowest, that is

$$
\min J=\min G(E k, E q) \text { s.t. }\left\{\begin{array}{l}
M b\left(x_{0}, y_{0}, z_{0} ; t\right) \geq M b_{0} \\
E k \leq E k_{0} \\
E q \leq E q_{0}
\end{array}\right.
$$

where, $\Omega\left(x_{0}, y_{0}, z_{0}\right)$ refers to the coordinate in the target area, $E k_{0}$ is the set of all the types of the smoke release equipment, and $E q_{0}$ is the set of the amounts of various smoke release equipment.

The calculation method of the smoke release optimal model. Since Equation (3) contains inequality, it is impossible to be solved directly. Thus, a new set of solutions are needed. Apply the penalty function method architecture to the smoke release optimal model

$$
J_{\text {new }}=G(E k, E q)+\gamma \sum_{j=1}^{N} w\left[M b\left(x_{0}, y_{0}, z_{0} ; t\right)-M b_{0}\right]
$$

where $w(p)=\left\{\begin{array}{lc}p^{2} & p \geq 0 \\ p^{2} \exp \left(-p^{2} / \beta\right) & p<0\end{array}\right.$, where, $\gamma, \beta$ are coefficients, which can be adjusted as it requires.

Use the adjoint method to solve this model, and the optimal smokescreen release schemes are thereupon worked out.

System integration. The smokescreen release precision control system is a complex system of multiple hardware and software platforms, multiple operation systems and multiple numeric models. Its framework is shown in Figure 2 as follows, mainly divided into three parts: 




Figure 2. The integration of the smokescreen release precision control system.

(1) The hardware platform is divided into three levels: the super computers, the application servers and the display computers. The super computers are used for the numerical forecasting models, such as WRF, the SDMx and the adjoint model of the SDMx; the application servers run the SROM and other calculation models based on the GIS technologies; the display computers on PCs, Tablets and so on.

(2) The operation systems include Linux and Windows. Linux mainly operates NCEP, WRF, SDMx and the adjoint model of SDMx, and so on. Windows is primarily used to operate the database, the middleware and the secondary analysis and demonstration models of the GIS system data.

(3) The integrated numerical models include the meteorological field forecasting, the smokescreen diffusion, the smokescreen release optimal control, and so on.

\section{Summary}

Facing the challenges on smokescreen precise release under the background of the information age, accelerating the theoretical framework establishment for the precision control and breaking through the critical technical bottlenecks in smokescreen precision control can address the problems that the existing crude smokescreen estimation models have in meeting the requirements of accurate decisions under complex conditions, and overcome the defects of the smokescreen application schemes, that is, separation from the actual situation and inflexibility in targeted smokescreen release, and thus further enhance the preciseness of the smokescreen protection. Therefore, it is very important to adopt the atmospheric numerical simulation to develop the theoretical framework of the smokescreen release precision control based on complex terrains and inverse the shielding targets.

\section{Acknowledgements}

This study was supported by the Natural Science Foundation of China Grant No. 41375154 and the R\&D Special Fund for Public Welfare Industry of meteorology (Grant GYHY201106033).

\section{References}

[1] Chen, H.P. (2002) Smokescreen Technical Basis. Ordnance Industry Press, Beijing.

[2] Searcy, C., Dean, K. and Stringer, W. (1998) PUFF: A Lagrangian Trajectory Volcanic Ash Tracking Model. Journal of Volcanology and Geothermal Research, 80, 1-16.

[3] Huang, S.X., Liu, P., Chen, H.P., et al. (2010) Numerical Simulation and Experimental Comparison on Atmospheric Pollution Chemical Accident Hazard Predicting (CDM). Acta Scientiarum Naturalium Universitatis Pekinensis, 47, 3538.

[4] Bartzis, J., Varvayanni, M., Venetsanos, A., et al. (1999) ADREA-Prog: A Three-Dimensional Finite Volumes. Mesoscale Meteorological Prognostic Model. Report RODOS (WG2)-TN (99)-02. 
[5] Yao, R.T., Hao, H.W., Huang, J., et al. (2006) A Real-Time System TW-NAOCAS of Accident Consequence Assessment for Tianwan NPP. Radiation Protection Bulletin, 26, 1-9.

[6] Information on http://www.acronymfinder.com/Science-and-Medicine/CBNP.html

[7] Sullivan, T.J., Ellis, J.S., Foster, C.S., et al. (1993) Atmospheric Release Advisory Capability: Real-Time Modeling of Airborne Hazardous Materials. Bulletin of the American Meteorological Society, 74, 2343-2361.

[8] Imai, K., Chino, M., Ishikawa, H., et al. (1985) SPEEDI: A Computer Code System for the Real-Time Prediction of Radiation Dose to the Public Due to an Accidental Release. JAERI, 1297, 89.

[9] Huang, S.X., Liu, F., Li, H.M. and Zuo, Y. (2010) Numerical Study on the Chemical Hazard Aiming at the Security during 2008 Beijing Olympic Games. 2010 Second World Congress on Software Engineering (WCSE), Wuhan, 19-20 December 2010, 94-97.

[10] Huang, S.X., Wang, X.M., Fu, T.B., et al. (2011) An Integrated Approach on Predicting, Warning and Optimized Controlling System Based on GIS for Nuclear Accident Hazard. The 2011 International Conference on Computers, Communications, Control and Automation (CCCA 2011), IEEE, Hong Kong, 301-305.

[11] Huang, S.X., Yang, W.D., Zhou, J.Y., et al. (in press) Hazard Assessment for Japanese Abandoned Chemical Weapons Destruction Based on Local-scale Atmospheric Dispersion Simulation. Applied Mechanics and Materials.

[12] Winiarek, V., Bocquet, M., Duhanyan, N., et al. (2014) Estimation of the Caesium-137 Source Term from the Fukushima Daiichi Nuclear Power Plant Using a Consistent Joint Assimilation of Air Concentration and Deposition Observations. Atmospheric Environment, 82, 268-279.

[13] Stohl, A., Seibert, P., Arduini, J., et al. (2009) An Analytical Inversion Method for Determining Regional and Global Emissions of Greenhouse Gases: Sensitivity Studies and Application to Halocarbons. Atmospheric Chemistry and Physics, 9, 1597-1620.

[14] Huang, S.X., Zeng, Q.C., Hu, F., et al. (in press) An Optimal Control Theory Framework for Nuclear and Chemical Disaster Emergency Based on the Natural Cybernetics. Journal of University of Chinese Academy of Sciences.

[15] Liu, F., Hu, F. and Zhu, J. (2005) Adjoint Method for the Optimum Planning of Industrial Pollutant Sources. Science in China Series D: Earth Sciences, 48, 1270-1279.

[16] Liu, F., Zhang, Y.H. and Hu, F. (2005) Adjoint Method for Assessment and Reduction of Chemical Risk in Open Spaces. Environmental Modeling \& Assessment, 10, 331-339.

[17] Liu, F., Zhu, J., Hu, F., et al. (2007) An Optimal Weather Condition Dependent Approach for Emission Planning in Urban Areas. Environmental Modelling \& Software, 22, 548-557.

[18] Zeng, Q.C. (1996) Natural Cybernetics. Bulletin of Chinese Academy of Sciences, 1, 16-21. 